\title{
Depressive symptoms, age at diagnosis and a clinically defined metabolic syndrome as independent correlates of glycemic control in patients with type 2 diabetes mellitus
}

\begin{abstract}
Objective: The findings concerning the association of metabolic syndrome (MetS) and depression with glycemic control are controversial. Recently, age at type 2 diabetes mellitus (T2DM) diagnosis has attracted particular attention but the relevant findings are limited. This study aimed to further assess the association between age at T2DM diagnosis, depressive symptoms and MetS with glycemic control in T2DM patients.
\end{abstract}

Methods: In 116 T2DM patients we assessed disease-related and MetS-related variables as well as depressive symptom severity (Center for Epidemiological Studies-Depression scale - CES-D). Glycemic control, as assessed by glycated hemoglobin (HBA1c) levels, was the dependent variable.

Results: Suboptimal HBA1c levels, i.e. $7 \%$ ( $53 \mathrm{mmol} / \mathrm{mol}$ ) or higher, were found in $35.3 \%$ of the patients. Elevated CES-D scores indicative of clinically relevant depressive symptoms $(C E S-D \geq 16)$ or possible major depression (CES-D $>23$ ) where found in $31.9 \%$ and $11.2 \%$ of the patients, respectively. Younger age at T2DM diagnosis $(\mathrm{p}=0.004)$, greater treatment intensity $(\mathrm{p}=0.009)$, higher levels of depressive symptoms $(\mathrm{p}=0.011)$ and the presence of a clinically defined MetS $(\mathrm{p}=0.012)$ were the variables independently associated with glycemic control in multiple regression analysis after adjusting for sex, education, marital status, family history of diabetes and disease duration.

Conclusion: In T2DM patients, the linear association found between depressive symptom severity and glycemic control points to the need of depressive symptoms being timely addressed. Younger age at T2DM onset may also be an important correlate of suboptimal glycemic control, independent of disease duration. Finally, classification of MetS in T2DM by clinical severity might help delineate its impact on glycemic control.

Keywords: diabetes mellitus, hba1c, age at onset, disease duration, depression, metabolic syndrome, biopsychosocial
Volume 2 Issue 6 - 2015

Elias Andreoulakis, ' Thomas Hyphantis, ${ }^{2}$ Apostolos lacovides,' Dimitrios Kandylis,' John G Yovos, ${ }^{3}$ loannis Nimatoudis'

'Department of Psychiatry,Aristotle University of Thessaloniki, Greece

2Department of Psychiatry, University of loannina, Greece ${ }^{3}$ Department of Internal Medicine, Aristotle University of Thessaloniki, Greece

Correspondence: Thomas Hyphantis, Department of Psychiatry, Medical School, University of loannina, loannina 45II0, Greece, Tel 302651007322

Email thomashyphantis@outlook.com

Received: April 05, 2015 | Published: May 05, 2015
Abbreviations: BMI, body mass index; CES-D, centre for epidemiological studies-depression scale; DM, diabetes mellitus; EM, expectation-maximization; HBA1c, glycated haemoglobin; HDL-C, high-density lipoprotein cholesterol; IDF, international diabetes federation; LDL-C, low-density lipoprotein cholesterol; MCAR, missing completely at random; MetS, metabolic syndrome; MNAR, missing not at random; NCEP-ATP-III, national cholesterol education programme-adult treatment panel iii; T2DM, type 2 diabetes mellitus; WHO, world health organization

\section{Introduction}

Diabetes mellitus (DM) affects nearly $6 \%$ of the population and long-term maintenance of optimal glycemic control has been established as a major therapeutic goal, ${ }^{2}$ since it has been prospectively associated with decreased risk of diabetic complications. ${ }^{3,4}$ Therefore, the identification of the factors associated with optimal glycemic control is considered a research priority.

Depression is approximately twice as frequent in patients with DM compared to individuals without DM. ${ }^{5,6}$ Furthermore, depression among patients with DM seems to be more prevalent in low- and middle-income countries than in high-income countries, although the relevant studies are limited. ${ }^{7}$ The risk for incident depression in patients with DM is estimated at approximately $1.25 .^{8,9}$ Comorbid depression in patients with DM has been associated with compromised adherence to self-care and adverse health outcomes. ${ }^{8,10}$ The findings concerning the association between depression and glycemic control in patients with type 2 DM (T2DM) remain controversial; a meta-analysis, ${ }^{11}$ a number of cross-sectional ${ }^{12,13}$ and longitudinal ${ }^{14-17}$ studies as well as findings of clinical trials ${ }^{18}$ argue in favor of such an association. However, there are also studies that did either found no association, ${ }^{19}$ or found an association that did not hold after controlling for factors such as social support and social comparison. ${ }^{20}$ In addition, there are longitudinal studies that found no association between improvement in depressive symptoms and improvement in HBA $1 \mathrm{c},{ }^{21-23}$ while others found an association between depressive symptoms at baseline and HBA1c at follow-up, which did not hold after controlling for baseline HBA1c. ${ }^{24}$

Age at T2DM onset has drawn attention only recently, since previous studies focused mainly on disease duration. An alarming trend to a declining mean age at T2DM onset has been reported. ${ }^{25}$ Earlier age at onset has been found to be linearly associated with higher HBA1c in a recent study, ${ }^{26}$ whereas a relatively earlier age at onset, i.e. before 65 years of age, was also found to be associated with higher HBA1c levels. ${ }^{27}$ 
The metabolic syndrome (MetS) represents a constellation of factors, namely central obesity, hyperglycemia, dyslipidemia and hypertension, associated with increased risk for cardiovascular disease. ${ }^{28}$ The prevalence of MetS in T2DM patients varies widely across studies, probably reflecting the divergence in the diagnostic criteria applied as well as cross-cultural differences. The former is illustrated in a study among 700 adults with T2DM in India, ${ }^{29}$ where the prevalence of MetS was $28 \%, 45.8 \%$ and $57.7 \%$ following WHO, ${ }^{30}$ NCEP-ATP-III ${ }^{31}$ and IDF criteria, ${ }^{32}$ respectively. Besides inconsistencies due to different criteria applied, the prevalence of MetS has been found to vary by country; for instance, MetS was found in $29.2 \%$ among 448 Asian Indian T2DM patients, ${ }^{33}$ in $51.2 \%$ among 5829 Chinese T2DM patients ${ }^{34}$ and in $70 \%$ among 1314 T2DM patients in Italy, ${ }^{35}$ according to NCEP-ATP III criteria. The findings concerning the association between the presence of MetS and glycemic control are inconclusive so far with studies arguing either in favor ${ }^{34,35}$ or against ${ }^{36}$ such an association or identifying an association between MetS and higher HBA1c levels in univariate but not multivariate analysis. ${ }^{37}$

The present study aimed to further assess the association between glycemic control and depressive symptoms and/or MetS as well as the association between glycemic control and age at T2DM diagnosis. With regard to the association between glycemic control and depressive symptoms, we sought to investigate whether such an association would hold in a multivariate analysis after controlling for additional factors that have not been broadly addressed, namely age at T2DM diagnosis, family history of diabetes and the presence of MetS. Concerning the association between age at T2DM diagnosis and glycemic control, we aimed to provide further support and extend the findings of previous studies by investigating whether a linear inverse association between age at T2DM diagnosis and HBA1c would hold after controlling for T2DM duration, the presence of MetS and depressive symptoms severity.

\section{Materials and methods}

\section{Participants}

The sample derived from the Diabetological outpatient unit of the 1st Internal Medicine Department of AHEPA General Hospital, Thessaloniki, Greece. Inclusion criteria were: established diagnosis of T2DM and ability to fully understand and fill-in the selfreport questionnaires. Exclusion criteria were: presence of current psychiatric morbidity and/or use of psychotropic medication, since psychotropic agents might affect both glycemic control and the scoring in the psychometric scales, thus obscuring the associations under investigation. One hundred and fifty nine patients were invited. After thorough explanation of the research protocol, 126 patients agreed to participate and provided informed consent (response rate: $79 \%$ ). After taking exclusion criteria into account, the effective sample consisted of 116 patients with T2DM. All procedures followed were in accordance with the Helsinki Declaration concerning human experimental research. The study protocol was approved by the Bioethics Committee of the Aristotle University of Thessaloniki.

The descriptive characteristics of the sample are presented in Table 1 . The two sexes were almost equally distributed in the sample. Participants' age ranged from 26 to 80years (median: 62years). The majority was married and half of them had received primary education. A positive family history of T2DM was present in $57.8 \%$ of the patients. Median age at T2DM diagnosis was 51.5 years and median duration of T2DM was 7.9 years. Nearly two thirds of the patients were currently treated with oral hypoglycemic agents. Median BMI was $28.8 \mathrm{~kg} / \mathrm{m}^{2}$ Obesity, dyslipidemia and hypertension were found in $43.1 \%, 41.5 \%$ and $55.2 \%$, respectively. According to the clinical criteria applied, MetS was present in $45.7 \%$ of the patients. Median HBA1c was $6.5 \%(47.5 \mathrm{mmol} / \mathrm{mol})$ and $35.3 \%$ of the patients had HBA1c $\geq 7 \%(53 \mathrm{mmol} / \mathrm{mol})$. Median score in CES-D scale was 11 ; almost one in three patients $(31.9 \%)$ had a score of 16 or higher in CES-D, indicative of clinically relevant depressive symptoms, whereas $11.2 \%$ had a score $>23$, indicative of possible major depression.

Table I Sample characteristics (demographics, diabetes-related and metabolic syndrome-related variables) and depressive symptoms assessed with CES-D scale

\begin{tabular}{|c|c|}
\hline \multirow{2}{*}{\multicolumn{2}{|c|}{$\operatorname{Sex}(N, \%)$}} \\
\hline Male & \\
\hline Male & $60(51.7 \%)$ \\
\hline Female & $56(48.3 \%)$ \\
\hline Age, Years (mean $\pm S D$ ) & $60.63 \pm 10.76$ \\
\hline \multicolumn{2}{|l|}{ Education Level $(\mathrm{N}, \%)$} \\
\hline Low (Primary) & $61(52.6 \%)$ \\
\hline Medium (Secondary) & $34(29.3 \%)$ \\
\hline High (Tertiary) & $21(18.1 \%)$ \\
\hline \multicolumn{2}{|l|}{ Family Status (N, \%) } \\
\hline Having a Spouse & $96(82.8 \%)$ \\
\hline Not having a Spouse & $20(17.2 \%)$ \\
\hline \multicolumn{2}{|l|}{ Family History of T2DM (N, \%) } \\
\hline No & 49 (42.2\%) \\
\hline Yes & $67(57.8 \%)$ \\
\hline Age at T2DM Diagnosis, Years (mean \pm SD) & $50.69 \pm 10.63$ \\
\hline T2DM Duration, Years (mean $\pm S D$ ) & $9.96 \pm 7.52$ \\
\hline \multicolumn{2}{|l|}{ Treatment Intensity (N, \%) } \\
\hline Low (Diet only) & $9(7.8 \%)$ \\
\hline Medium (Hypoglycemic pills) & $75(64.7 \%)$ \\
\hline High (Insulin) & $32(27.6 \%)$ \\
\hline $\mathrm{BMI}, \mathrm{kg} / \mathrm{m} 2(\mathrm{mean} \pm \mathrm{SD})$ & $29.92 \pm 5.80$ \\
\hline \multicolumn{2}{|l|}{ Obesity, i.e. $\mathrm{BMl}>30$ kg/m2 (N, \%) } \\
\hline No & $66(56.9 \%)$ \\
\hline Yes & $50(43.1 \%)$ \\
\hline \multicolumn{2}{|l|}{ Dyslipidemia Under Treatment (N, \%) } \\
\hline No & $68(58.6 \%)$ \\
\hline Yes & $48(4 I .4 \%)$ \\
\hline \multicolumn{2}{|l|}{ Hypertension Under Treatment (N, \%) } \\
\hline No & $52(44.8 \%)$ \\
\hline Yes & $64(55.2 \%)$ \\
\hline \multicolumn{2}{|l|}{ Metabolic Syndrome (MetS) (N, \%) } \\
\hline No & $63(54.3 \%)$ \\
\hline Yes & $53(45.7 \%)$ \\
\hline HBAIc, \% (mean \pm SD) & $6.73 \pm 1.39$ \\
\hline CES-D Score (mean \pm SD) & $|2.8| \pm 8.32$ \\
\hline
\end{tabular}

\section{Procedures}

Data concerning demographics, diabetes-related and MetS-related variables, as well as depressive symptoms were gathered. Demographic variables included sex, age, family status and educational level. Diabetes-related variables included HBA1c, age at T2DM diagnosis, T2DM duration and family history of T2DM and treatment modality which reflected treatment intensity. Diet, oral hypoglycemic agents and insulin, represented low, moderate and high treatment intensity, respectively. HBA1c was treated as a continuous variable for the purposes of the analysis. In addition, for the descriptive part of the analysis, an HBA1c value of $7 \%(53 \mathrm{mmol} / \mathrm{mol})$ or higher was 
regarded as indicative of suboptimal glycemic control. ${ }^{2}$ MetS-related variables included body mass index (BMI), obesity, hypertension and dyslipidemia. Obesity was defined as BMI $>30 \mathrm{~kg} / \mathrm{m}^{2}$, whereas hypertension and dyslipidemia were considered as present so long as the patients had a relevant diagnosis and were currently under appropriate medication. For the purpose of the analysis, obesity, hypertension and dyslipidemia were not treated as separate variables but were combined in a composite categorical variable, namely MetS. MetS was considered as present as long as at least two among the following three, namely obesity, hypertension and dyslipidemia, were present. Our criteria are close to IDF ${ }^{32}$ and NCEP-ATP III, ${ }^{31}$ with slight modifications. In specific, central obesity was assessed according to BMI instead of waist circumference, whereas the assessment of hypertension and dyslipidemia was not based on laboratory measurements (i.e. Blood pressure, Triglycerides, HDL-C levels) but on the presence of established clinical nosology (i.e. Diagnosis and appropriate treatment of hypertension and/or dyslipidemia). In that sense, our criteria were stricter - thus referring to a 'clinically defined MetS' - potentially leading to an underestimation of MetS in comparison to IDF or NCEP-ATP III criteria. For instance, in those patients with both high triglycerides and low HDL-C, dyslipidemia alone - besides T2DM - would satisfy the criteria for MetS according to IDF and NCEP-ATP III. In contrast, our clinical definition of MetS, required dyslipidemia to be accompanied by at least one more clinical condition (hypertension under treatment and/or obesity) in order for MetS to be regarded as present.

Depressive symptoms were assessed with the Center for Epidemiological Studies - Depression (CES-D) scale. CES-D is a 20 -item questionnaire that assesses depressive symptoms over the previous week. Items are rated in a 4-point Likert scale (0-3) and the total score ranges from 0 to 60 . Higher scores indicate greater severity of depressive symptoms. In a recent systematic review, CES-D was identified as the second most popular screening tool, applied in $21 \%$ of the studies among patients with DM..$^{38}$ Moreover, a comparative study found that CES-D score was the best predictor of depression in patients with T2DM with the highest ability to discriminate between depression and other non-depressive symptoms. ${ }^{39}$ CES-D has been validated in Greek yielding a Cronbach's alpha of 0.95. A cut-off level of $23 / 24$ was identified as yielding both sensitivity and specificity values above 0.90 concerning the detection of major depression. ${ }^{40}$ Therefore, we considered a score of above 23 in CES-D scale as indicative of possible major depression. We also applied the cut-off level of 16 or higher as indicative of clinically relevant depressive symptoms, as established in the literature. ${ }^{41,42}$ The aforementioned cutoffs were used for the descriptive part of the study. For the purpose of the main analysis, CES-D score was treated as a continuous variable. Cronbach's alpha in our dataset of patients with complete data on CES-D scale $(\mathrm{N}=89)$ was 0.87 .

\section{Statistical analysis}

Exploratory and preliminary analysis - Treatment of missing values: Unfortunately, the presence of missing values concerning CES-D scale and HBA1c measurements on the very day of assessment was not avoided. The amount of complete data cases for the variables of interest were as follows: CES-D score: 89 , HBA1c: 88 . Listwise deletion of missing data cases would result in a limited sample size $(\mathrm{N}=69)$ that would not afford the intended multivariate analysis with 9 independent variables. Thus, aiming at increasing the sample size available for the intended multivariate analysis and maximizing the power of the analysis, a missing values analysis was performed and Expectation-Maximization (EM) procedure was applied in order for the missing data to be imputed. In order for EM to be applicable, we tested whether the possibility of data Missing Not At Random (MNAR) would not hold. Data MNAR would be the case if, for instance, patients with higher levels of depressive symptoms indeed avoided providing data on CES-D scale, or patients with higher HBA1c avoided taking the blood test on the day of the assessment. As a first indication for the applicability of EM procedure Little's test of data Missing Completely At Random (MCAR) was non-significant $(p=0.876)$. In order to further verify the applicability of EM and the validity of the results it produced we performed a post hoc analysis, i.e. a comparison between the original and the imputed (obtained by EM) values of HBA1c and CES-D scores by means of two separate independent samples t-tests between originally complete data vs originally missing data (finally imputed by EM) dataset for each of the variable of interest, namely HBA1c or CES-D score. In case any significance difference between the two datasets emerged, the data would be MNAR. We found that mean HBA $1 \mathrm{c}$ values were comparable between the originally complete data and the originally missing data (finally imputed by EM) dataset ( $6.72 \pm 1.53$ vs $6.75 \pm 0.81$; $=0.881$ ) and the same was true regarding mean CES-D scores (12.56 \pm 9.35 vs $13.55 \pm 3.85 ; \mathrm{p}=0.426$ ). Therefore, the possibility of data MNAR could again be dropped and the applicability of EM was post hoc verified.

\section{Main analysis}

Age was not included in the analysis due to strong intercorrelations and multicollinearity; age was strongly correlated with both age at T2DM diagnosis $(\mathrm{r}=0.754)$ and T2DM duration $(\mathrm{r}=0.366)$ as expected, since T2DM duration is a function of age and age at T2DM (T2DM duration $=$ age - age at $\mathrm{T} 2 \mathrm{DM}$ diagnosis). Thus, a linear multiple regression was performed with HBA1c as the dependent variable and 9 independent variables, namely sex, educational level, family status, family history of DM, age at T2DM diagnosis, T2DM duration, treatment intensity, presence of MetS and CES-D score.

\section{Results}

As a preliminary step, we investigated for any multicollinearity issues. For this purpose, we first inspected the univariate correlations among the independent variables. As shown in Table 2, no strong correlations among the predictors were present; the highest correlation coefficient was observed between T2DM duration and treatment intensity (Spearman $\mathrm{rho}=0.42$, reflecting a small to moderate association). Multicollinearity was further explored within the multiple regression analysis and no multicollinearity issues emerged, since all tolerance values were higher than 0.5 and all variance inflation factor (VIF) values were lower than 2 .

In the main linear multiple regression analysis, 9 independent variables were entered in the model and four of them were significantly associated with higher HBA1c values. The model was significant $(p<0.001)$ with an $F(9,106)$ equal to 6.827 and accounted for $36.7 \%$ of HBA1c variance. As for the independent correlates of HBA1c, younger age at T2DM diagnosis $(\mathrm{p}=0.004)$, greater treatment intensity $(p=0.009)$, higher levels of depressive symptoms $(p=0.011)$ and the presence of a clinically defined MetS $(p=0.012)$ were independently associated with higher HBA1c levels.

Given than treatment intensity is bidirectionally associated with HBA1c levels (as poor glycemic control often results in intensification of treatment regimen), we thought that HBA1c would be as likely to determine treatment intensity as to be determined by it. Thus, we performed another multiple regression analysis without including "treatment intensity" among the independent variables. The 8-variable 
model was again significant $(\mathrm{p}<0.001)$ with an $\mathrm{F}(8,107)$ equal to 6.418 and accounted for $32.4 \%$ of HBA1c variance. Alongside with the same independent correlates as before (i.e. younger age at T2DM diagnosis, presence of a clinically defined MetS and higher levels of depressive symptoms), longer T2DM duration was also found to be independently associated with higher HBA1c levels $(\mathrm{p}=0.018)$.

The results of the two multiple regression analyses are presented in Table 3. As stated above, no remarkable multicollinearity issues that might bias the regression models emerged in the multiple regression analyses; specifically, for the 9-predictor model, the lowest tolerance value observed was 0.696 (accordingly, the highest VIF value was 1.436 ) for the variable 'treatment intensity'. Similarly, in the 8-predictor model, the lowest tolerance value observed was 0.751 (accordingly, the highest VIF value was 1.332) for the variable of 'age at T2DM diagnosis'.

Table 2 Univariate associations among independent variables and between each independent variable and the dependent variable (HBAIC)

\begin{tabular}{|c|c|c|c|c|c|c|c|c|c|c|}
\hline & HBAIc & $\begin{array}{l}\text { Sex } \\
\text { (Female) }\end{array}$ & $\begin{array}{l}\text { Education } \\
\text { Level }\end{array}$ & $\begin{array}{l}\text { Family } \\
\text { Status (with } \\
\text { spouse) }\end{array}$ & $\begin{array}{l}\text { Family } \\
\text { History of } \\
\text { Diabetes }\end{array}$ & $\begin{array}{l}\text { Age at } \\
\text { T2DM } \\
\text { Diagnosis }\end{array}$ & $\begin{array}{l}\text { T2DM } \\
\text { Duration }\end{array}$ & $\begin{array}{l}\text { Treatment } \\
\text { Intensity }\end{array}$ & MetS & $\begin{array}{l}\text { CES-D } \\
\text { Score }\end{array}$ \\
\hline HBAIc & & 0.074 & -0.141 & -0.108 & $-0.04 I$ & -0.285 & $-0.319 * *$ & $0.422 * *$ & $0.297 * *$ & $0.315 * *$ \\
\hline Sex (Female) & 0.074 & & $-0.280 * *$ & $-0.244 * *$ & 0.023 & -0.112 & -0.049 & $-0.07 \mid$ & 0.014 & $0.360 * *$ \\
\hline Education Level & $-0.14 \mid$ & $-0.280 * *$ & & 0.036 & -0.016 & $-0.20 I *$ & -0.025 & -0.121 & $-0.226 *$ & -0.301 \\
\hline $\begin{array}{l}\text { Family Status } \\
\text { (with spouse) }\end{array}$ & -0.108 & $-0.244 * *$ & 0.036 & & -0.021 & 0.058 & 0.028 & 0.09 & 0.052 & $-0.269 * *$ \\
\hline $\begin{array}{l}\text { Family History of } \\
\text { Diabetes }\end{array}$ & -0.041 & 0.023 & -0.016 & -0.021 & & -0.029 & -0.124 & -0.162 & 0.049 & 0.096 \\
\hline $\begin{array}{l}\text { Age at T2DM } \\
\text { Diagnosis }\end{array}$ & -0.285 & -0.112 & $-0.20 I *$ & 0.058 & -0.029 & & $-0.306 * *$ & -0.112 & 0.165 & -0.074 \\
\hline T2DM Duration & $-0.319 * *$ & -0.049 & -0.025 & 0.028 & -0.124 & $-0.306 * *$ & & $0.426 * *$ & 0.047 & 0.105 \\
\hline $\begin{array}{l}\text { Treatment } \\
\text { Intensity }\end{array}$ & $0.422 * *$ & $-0.07 \mid$ & -0.121 & 0.09 & -0.162 & -0.112 & $0.426 * *$ & & $0.323 * *$ & 0.106 \\
\hline MetS & $0.297 * *$ & 0.014 & $-0.226 *$ & 0.052 & 0.049 & 0.165 & 0.047 & $0.323 * *$ & \multirow{2}{*}{0.066} & \multirow[t]{2}{*}{0.066} \\
\hline CES-D Score & $0.315^{* *}$ & $0.360 * *$ & -0.301 & $-0.269 * *$ & 0.096 & -0.074 & 0.105 & 0.106 & & \\
\hline
\end{tabular}

Note: Data represent Spearman correlation coefficients. Statistical significance is indicated with asterisks as follows: ${ }^{* * *}: \mathrm{p}<0.00 \mathrm{I} ;{ }^{* *}: \mathrm{p}<0.0 \mathrm{I} ; *^{*} \mathrm{p}<0.05$

For dichotomous variables (i.e. sex, family status and family history of diabetes and presence of MetS) the coefficient represents point-biserial correlation

Table 3 Liner multiple Regression analysis to identify the factors independently associated with glycemic control, the latter as reflected by HBA Ic values

\begin{tabular}{|c|c|c|c|c|}
\hline \multirow[b]{2}{*}{ Variable } & \multicolumn{2}{|c|}{ Model I (9 Regressors) } & \multicolumn{2}{|c|}{ Model 2 (8 Regressors) } \\
\hline & Standardized beta & $\mathrm{P}$ & Standardized beta & $P$ \\
\hline Sex (Female) & -0.077 & 0.384 & -0.093 & 0.307 \\
\hline Education Level & -0.08 & 0.371 & -0.09 & 0.326 \\
\hline Family Status (with spouse) & -0.078 & 0.336 & -0.065 & 0.436 \\
\hline Family History of Diabetes & -0.014 & 0.864 & -0.054 & 0.507 \\
\hline Age at T2DM Diagnosis & -0.263 & 0.004 & -0.272 & 0.004 \\
\hline Treatment Intensity & 0.248 & 0.009 & \multicolumn{2}{|c|}{ Not included in the model } \\
\hline Presence of Metabolic Syndrome & 0.219 & 0.012 & 0.294 & $\begin{array}{l}\text { in the model } \\
0.001\end{array}$ \\
\hline Depressive Symptoms (CES-D score) & 0.229 & 0.011 & 0.237 & 0.011 \\
\hline \multirow[t]{2}{*}{ Regression Statistics } & \multicolumn{2}{|c|}{$F(9,106)=6.827 ; p<0.001$} & \multicolumn{2}{|c|}{$F(8,107)=6.418 ; p<0.001$} \\
\hline & \multicolumn{2}{|c|}{$\mathrm{R} 2=0.367 ;$ adjusted $\mathrm{R} 2=0.3 \mathrm{I} 3$} & \multicolumn{2}{|c|}{$\mathrm{R} 2=0.324 ;$ adjusted $\mathrm{R} 2=0.274$} \\
\hline
\end{tabular}

\section{Discussion}

The results of the present study showed that depressive symptoms are common in Greek patients with DM, as in other populations. A percentage of $31.9 \%$ of the patients presented scores of 16 or higher in CES-D scale, indicative of clinically relevant depressive symptoms and $11.2 \%$ presented scores above 23 , indicative of possible major depression. These findings are in line with the prevalence rates reported for T2DM patients in a relevant meta-analysis ${ }^{6}$ regarding clinically significant depressive symptoms $(32.9 \%$ as assessed by self-report questionnaires) and for major depression determined by diagnostic interviews $(10.9 \%)$. To the best of our knowledge, this is the first study to address depressive symptoms in patients with T2DM in Greece, so no comparison with relevant studies in our country was applicable.

We also found that $57.8 \%$ of our patients had a positive family history of DM, similarly to what has been found (53.6\%) in another study in Greece among 1473 patients with T2DM. ${ }^{43}$ In line with the results of this study, we also found no association between family history of diabetes and HBA1c levels.

Present findings showed a $45.7 \%$ prevalence of a clinically defined MetS. Although this rate falls within the aforementioned range reported in the literature, it is significantly lower than the $64.8 \%$ prevalence reported by another study in Greece. ${ }^{44}$ This discrepancy might be due to the definition of MetS in the present study, which reflected a clinical definition of MetS based on already established clinical nosology rather than a broader definition of MetS based on laboratory data. In addition, the assessment of obesity, which was based here on BMI, may reflect a less sensitive measure compared to waist circumference. In support of this explanation, abnormal waist circumference was found in $55.2 \%$ of the patients in the aforementioned study ${ }^{44}$ whereas obesity was found in $43.1 \%$ of the present sample. Hypertension rates were comparable ( $59.5 \%$ vs $55.2 \%)$. 
The results of the present study confirmed the linear association between depressive symptom severity and HBAlc in line with the results of the only meta-analysis performed so far ${ }^{11}$ and a substantial body of evidence. Given that are also exist controversial findings and in order to shed further light to the discrepancies across studies we conducted additional analyses with HBA1c and/or CES-D score treated as categorical variables (results available upon request). Of note, when CES-D score was treated as a categorical variable (applying the cut-off levels of 16 or 23), neither clinically relevant depressive symptoms nor possible major depression, respectively, was identified as independent correlates of HBA1c $(\mathrm{p}=0.071$ and $\mathrm{p}=0.140$, respectively). Similarly, when HBA1c was treated as a categorical variable - applying the cut-off level of $7 \%(53 \mathrm{mmol} /$ mol) -, depressive symptoms, either treated as a scale variable or as a categorical one, failed to be identified as independent correlate of HBA1c in the binomial logistic regression analyses performed ( $p=0.241, p=0.172$ and $p=0.421$, respectively). These findings may explain to some extent the discrepancy across relevant studies, in that the way depressive symptoms or HBA1c are treated within the statistical analysis (as a scale vs a categorical variable) might account for the observed inconsistencies. Nevertheless, the linear association found here between depressive symptoms and HBA1c carries important clinical implications, suggesting that depressive symptoms should be addressed timely, since they might exert a negative impact on glycemic control even before the development of a clinical depression. The exclusion of patients currently under psychotropic medication that might have interfered with both HBA1c levels and the presence of MetS as well as the scoring in CES-D scale, represented a strength of this study, enhancing a clearer investigation of the association between depressive symptoms and glycemic control after excluding potential confounders.

Our main new finding is that age at T2DM diagnosis was inversely associated with HBA1c levels. This finding confirms the findings of a previous study reporting an inverse linear association between age at $\mathrm{T} 2 \mathrm{DM}$ diagnosis and $\mathrm{HBA} 1 \mathrm{c}^{26}$ and extends its findings, as the aforementioned inverse linear association was found here to hold in multivariate analysis even after controlling for depressive symptoms severity and the presence of a clinically defined MetS. Age at diagnosis represents a field not adequately studied in T2DM, particularly with regard to its association with patients' well-being and quality of life alongside with the disease's course and outcome. Thus, a further exploration of the role of age at diagnosis represents a factor that carries important implications for future research as well.

Finally, we identified the presence of a clinically defined MetS as an independent correlate of HBA1c. Given that the presence of MetS is a marker of insulin resistance, the association between MetS and HBA1c is a more or less expected finding, though not consistently replicated across studies. The controversy with previous findings concerning the association between MetS and glycemic control ${ }^{36,37}$ might partly be due to the broadness as well as the divergence of criteria applied to assess MetS in T2DM. The findings of the present study, focusing on a clinically defined MetS, might contribute to shedding further light to the association between MetS and HBA1c.

There are some limitations in this study that have to be taken into account. Its cross-sectional design does not allow inferences about causality to be drawn, particularly concerning the association of depressive symptoms with glycemic control. The small sample size represents another limitation of this study. Although it was adequate for the intended multivariate analysis with 9 independent variables, it cannot guarantee the representativeness of the sample. In addition, we acknowledge that representativeness of the sample and generalizability of our findings might also be limited by the fact that the sample was drawn from patients attending a tertiary Diabetological outpatient unit. Although it is likely that many of our results would apply to patients with T2DM seen in other outpatient settings (especially since our results are consistent with studies in T2DM patients samples derived from different medical settings), the possibility that Diabetological unit attenders have different profiles could produce some selection bias. Glycemic control, for example, was quite good in the present sample, with the majority of patients having HBA1c $<7 \%(53 \mathrm{mmol} / \mathrm{mol})$, probably reflecting the medical service's potential (a tertiary hospital outpatient unit specialized in $\mathrm{DM})$. Another concern is that patients with relatively worse glycemic control (i.e. higher HBA1c values) might have been more reluctant to participate in the study. However, this seems less likely as indicated by the results of the post hoc analysis between the dataset with originally complete vs originally missing (later imputed by EM) HBA1c values. Finally, the presence of missing data was another limitation, which, however, was adequately dealt with by means of EM procedure, the applicability of which was verified both ad hoc and post hoc.

This study may have important implications for future research. It provides support to a recently growing body of evidence highlighting the role of age at T2DM diagnosis with regard to glycemic control and disease course. The finding of a linear association between age at T2DM diagnosis and glycemic control make the former, i.e. age at diagnosis, a significant factor deserving attention throughout a broad age spectrum not limited to certain age groups (e.g. children, adolescents, young adults or elderly patients above 65 ). We found that earlier T2DM onset is associated with higher HBA1c levels independent of disease duration. Additional multiple regression analyses perform including the interaction term "age at diagnosis $\mathrm{x}$ disease duration" as predictor variable in the 8- and 9-predictor models of Table 3 showed no statistically significant interaction for either model ( $p>.05)$; the results were similar as in Table 3 with only extremely minor deviations in the overall $R^{2}$ and adjusted $R^{2}$ values, standardized beta weights and individual predictor p-values. Therefore, it seems unlikely that earlier age at diagnosis is associated with higher levels of HBA1c by merely reflecting longer disease duration, i.e. chronicity. Although we acknowledge that what follows remains hypothetical since it could not be directly assessed in the present study, we consider it worth mentioning as it might represent a promising field for future research and practice.

Thus, concerning the association between age at diagnosis and HBA1c there might be another two possibilities, probably not mutually exclusive, in a biopsychosocial context. Firstly, earlier disease onset might reflect a biological vulnerability due to which insulin resistance may become more profound and manifest itself earlier. Secondly, earlier disease onset might particularly threaten psychological adaptation to DM diagnosis by imposing the burden of a chronic, demanding in terms of self-care and potentially dehabilitating disease early in life. Impaired psychological adaptation to DM diagnosis might, in turn, compromise long-term self-care and adherence to treatment regimen, thus leading eventually to higher HBA1c levels. Longitudinal studies investigating the association of age at T2DM diagnosis with T2DM course, progression and complications as well as with psychological adjustment, psychological distress and adherence to treatment regimens are needed, in order to enhance our understanding of the factors associated with the achievement and long-term maintenance of optimal glycemic control.

\section{Conclusion}

In T2DM patients, the linear association found between depressive symptom severity and glycemic control points to the need of depressive 
symptoms being timely addressed even before the development of clinical depression. Furthermore, younger age at T2DM onset emerges as an important correlate of suboptimal glycemic control, independent of disease duration. Thus, age at T2DM onset warrants particular attention and physicians should stay alert when treating patients with relatively early disease onset, addressing factors that might interfere with the psychosocial adaptation to the early diagnosis compromising glycemic control. Finally, given the association found between the presence of a clinically defined MetS and higher HBA1c levels, the classification of MetS in T2DM by clinical severity might help shedding further light to its impact on glycemic control.

\section{Acknowledgments}

None.

\section{Conflicts of interest}

Author declares there are no conflicts of interest.

\section{Funding}

None.

\section{References}

1. Mayor S. Diabetes affects nearly $6 \%$ of the world's adults. $B M J$. 2006;333(7580):1191

2. American Diabetes Association. Standards of medical care in diabetes--2014. Diabetes Care. 2014;37(Suppl 1):14-80.

3. Intensive blood-glucose control with sulphonylureas or insulin compared with conventional treatment and risk of complications in patients with type 2 diabetes (UKPDS 33). UK Prospective Diabetes Study (UKPDS) Group. Lancet. 352(9131):837-853.

4. The effect of intensive treatment of diabetes on the development and progression of long-term complications in insulin-dependent diabetes mellitus. The Diabetes Control and Complications Trial (DCCT) Group. N Engl J Med. 329(14):977-986.

5. Ali S, Stone MA, Peters JL, et al. The prevalence of co-morbid depression in adults with Type 2 diabetes: a systematic review and metaanalysis. Diabet Med. 2006;23(11):1165-1173.

6. Anderson RJ, Freedland KE, Clouse RE,et al. The prevalence of comorbid depression in adults with diabetes: a meta-analysis. Diabetes Care. 2001;24(6):1069-1078.

7. Mendenhall E, Norris SA, Shidhaye R, et al. Depression and type 2 diabetes in low- and middle-income countries: a systematic review. Diabetes Res Clin Pract. 2014;103(2):276-285.

8. Andreoulakis E, Hyphantis T, Kandylis D, et al. Depression in diabetes mellitus: a comprehensive review. Hippokratia. 2012;16(3):205-214.

9. Rotella F, Mannucci E. Diabetes mellitus as a risk factor for depression A meta-analysis of longitudinal studies. Diabetes Res Clin Pract. 2013;99(2):98-104.

10. Zuberi SI, Syed EU, Bhatti JA. Association of depression with treatment outcomes in Type 2 Diabetes Mellitus: a cross-sectional study from Karachi, Pakistan. BMC Psychiatry. 2011;11:27.

11. Lustman PJ, Anderson RJ, Freedland KE, et al. Depression and poor glycemic control: a meta-analytic review of the literature. Diabetes Care. 2000;23(7):934-942.

12. Gois C, Dias VV, Raposo JF, et al. Vulnerability to stress, anxiety and depressive symptoms and metabolic control in type 2 diabetes. BMC Res Notes. 2012;5:271.

13. Papelbaum M, Moreira RO, Coutinho W, et al. Depression, glycemic control and type 2 diabetes. Diabetol Metab Syndr. 2011;3(1):26.
14. Musselman DL, Ziemer DC, McNutt MD, et al. Depression, deficits in functional capacity, and impaired glycemic control in urban African Americans with type 2 diabetes. J Psychiatr Res. 2014;52:21-27.

15. Richardson LK, Egede LE, Mueller M, et al. Longitudinal effects of depression on glycemic control in veterans with Type 2 diabetes. Gen Hosp Psychiatry. 2008;30(6):509-514.

16. Dirmaier J, Watzke B, Koch U, et al. Diabetes in primary care: prospective associations between depression, nonadherence and glycemic control. Psychother Psychosom. 2010; 79(3):172-178.

17. Bot M, Pouwer F, de Jonge P, et al. Differential associations between depressive symptoms and glycaemic control in outpatients with diabetes. Diabet Med. 2013;30(3):115-122.

18. van der Feltz-Cornelis CM, Nuyen J, Stoop C, et al. Effect of interventions for major depressive disorder and significant depressive symptoms in patients with diabetes mellitus: a systematic review and meta-analysis. Gen Hosp Psychiatry. 2010;32(4):380-395.

19. Egede LE, Ellis C. The effects of depression on metabolic control and quality of life in indigent patients with type 2 diabetes. Diabetes Technol Ther. 2010;12(4):257-262

20. Arigo D, Smyth JM, Haggerty K, et al. The social context of the relationship between glycemic control and depressive symptoms in type 2 diabetes. Chronic Illn. 2014;11(1):33-43.

21. Gary TL, Baptiste-Roberts K, Crum RM, et al. Changes in depressive symptoms and metabolic control over 3 years among African Americans with type 2 diabetes. Int J Psychiatry Med. 2005;35(4):377-382.

22. Heckbert SR, Rutter CM, Oliver M, et al. Depression in relation to longterm control of glycemia, blood pressure, and lipids in patients with diabetes. J Gen Intern Med. 2010;25(6):524-529.

23. Georgiades A, Zucker N, Friedman KE, et al. Changes in depressive symptoms and glycemic control in diabetes mellitus. Psychosom Med. 2007;69(3):235-241.

24. Aikens JE, Perkins DW, Lipton B, et al. Longitudinal analysis of depressive symptoms and glycemic control in type 2 diabetes. Diabetes Care. 2009;32(7):1177-1181.

25. Pinhas-Hamiel O, Zeitler P. The global spread of type 2 diabetes mellitus in children and adolescents. J Pediatr. 2005;146(5):693-700.

26. Hsieh A, Ong PX, Molyneaux L, et al. Age of diabetes diagnosis and diabetes duration associate with glycated haemoglobin. Diabetes Res Clin Pract. 2014;104(1):1-4.

27. Berkowitz SA, Meigs JB, Wexler DJ. Age at type 2 diabetes onset and glycaemic control: results from the National Health and Nutrition Examination Survey (NHANES) 2005-2010. Diabetologia. 2013;56(12):2593-2600

28. Eckel RH, Grundy SM, Zimmet PZ. The metabolic syndrome. Lancet. 2005;365(9468):1415-1428

29. Yadav D, Mahajan S, Subramanian SK, et al. Prevalence of metabolic syndrome in type 2 diabetes mellitus using NCEP-ATPIII, IDF and WHO definition and its agreement in Gwalior Chambal region of Central India. Glob J Health Sci. 2013;5(6):142-155.

30. Alberti KG, Zimmet PZ. Definition, diagnosis and classification of diabetes mellitus and its complications. Part 1: diagnosis and classification of diabetes mellitus provisional report of a WHO consultation. Diabet Med. 1998;15(7):539-553.

31. National Cholesterol Education Program (NCEP) Expert Panel on Detection E, and Treatment of High Blood Cholesterol in Adults (Adult Treatment Panel III). Third Report of the National Cholesterol Education Program (NCEP) Expert Panel on Detection, Evaluation, and Treatment of High Blood Cholesterol in Adults (Adult Treatment Panel III) final report. Circulation. 2002;106(25):3143-3421. 
32. Alberti KG, Zimmet P, Shaw J. The metabolic syndrome--a new worldwide definition. Lancet. 2005;366(9491):1059-1062.

33. Das M, Pal S, Ghosh A. Family history of type 2 diabetes and prevalence of metabolic syndrome in adult Asian Indians. $J$ Cardiovasc Dis Res. 2012;3(2):104-108.

34. Luk AO, So WY, Ma RC, et al. Metabolic syndrome predicts new onset of chronic kidney disease in 5,829 patients with type 2 diabetes: a 5-year prospective analysis of the Hong Kong Diabetes Registry. Diabetes Care. 2008;31(12):2357-2361.

35. Bianchi C, Penno G, Daniele G, et al. The metabolic syndrome is related to albuminuria in Type 2 diabetes. Diabet Med. 2008;25(12):1412-1418.

36. Worawongprapa O. Glycemic control in diabetes with metabolic syndrome in community hospital.JMedAssoc Thai. 2008;91(5):641-647.

37. Cruz Del Castillo AH, Cordova Fernandez JA, Ramos Lagunes I, et al. Comparison of glycemic control in diabetic patients with and without metabolic syndrome. Rev Fac Cien Med Univ Nac Cordoba. 2013;70(4):193-200.

38. Roy T, Lloyd CE, Pouwer F, et al. Screening tools used for measuring depression among people with Type 1 and Type 2 diabetes: a systematic review. Diabet Med. 2012;29(2):164-175.
39. McHale M, Hendrikz J, Dann F, et al. Screening for depression in patients with diabetes mellitus. Psychosom Med. 2008;70(8):869-874

40. Fountoulakis K, Iacovides A, Kleanthous S, et al. Reliability, validity and psychometric properties of the Greek translation of the Center for Epidemiological Studies-Depression (CES-D) Scale. BMC Psychiatry. 2001;1:3.

41. Radloff LS. The CES-D Scale: A Self-Report Depression Scale for Research in the General Population. Appl Psychol Meas. 1977;1(3):385-401.

42. Beekman AT, Deeg DJ, Van Limbeek J, et al. Criterion validity of the Center for Epidemiologic Studies Depression scale (CES-D): results from a community-based sample of older subjects in The Netherlands. Psychol Med. 1997;27(1):231-235.

43. Papazafiropoulou A, Sotiropoulos A, Skliros E, et al. Familial history of diabetes and clinical characteristics in Greek subjects with type 2 diabetes. BMC Endocr Disord. 2009;9:12.

44. Panagiotakos DB, Pitsavos C, Chrysohoou C, et al. The epidemiology of Type 2 diabetes mellitus in Greek adults: the ATTICA study. Diabet Med. 2005;22(11):1581-1588. 